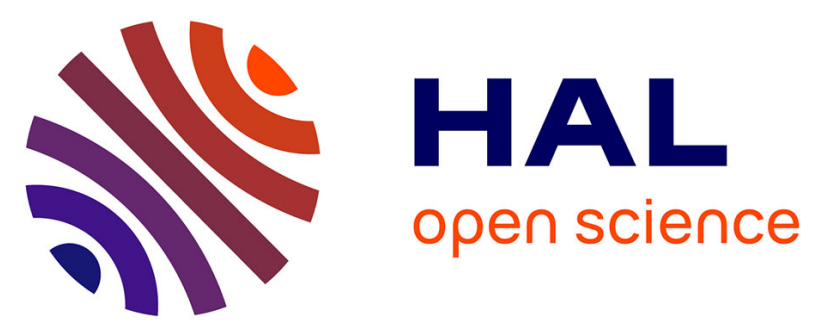

\title{
The Solar Wind Charge-Transfer X-Ray Emission in the 1/4 keV Energy Range: Inferences on Local Bubble Hot Gas at Low Z
}

\author{
Dimitra Koutroumpa, Rosine Lallement, J.C. Raymond, V. Kharchenko
}

\section{To cite this version:}

Dimitra Koutroumpa, Rosine Lallement, J.C. Raymond, V. Kharchenko. The Solar Wind ChargeTransfer X-Ray Emission in the 1/4 keV Energy Range: Inferences on Local Bubble Hot Gas at Low Z. The Astrophysical Journal, 2009, 696, pp.1517-1525. 10.1088/0004-637X/696/2/1517 . hal00379193

\section{HAL Id: hal-00379193 \\ https://hal.science/hal-00379193}

Submitted on 10 Jul 2020

HAL is a multi-disciplinary open access archive for the deposit and dissemination of scientific research documents, whether they are published or not. The documents may come from teaching and research institutions in France or abroad, or from public or private research centers.
L'archive ouverte pluridisciplinaire HAL, est destinée au dépôt et à la diffusion de documents scientifiques de niveau recherche, publiés ou non, émanant des établissements d'enseignement et de recherche français ou étrangers, des laboratoires publics ou privés. 


\title{
THE SOLAR WIND CHARGE-TRANSFER X-RAY EMISSION IN THE 1/4 keV ENERGY RANGE: INFERENCES ON LOCAL BUBBLE HOT GAS AT LOW Z
}

\author{
D. Koutroumpa ${ }^{1}$, R. Lallement ${ }^{1}$, J. C. Raymond ${ }^{2}$, and V. Kharchenko ${ }^{2}$ \\ ${ }^{1}$ UMR 7620, IPSL/Service d'Aéronomie, CNRS, Université de Versailles-Saint Quentin en Yvellines, Université Pierre et Marie Curie, \\ Verrières le Buisson, 91371, France \\ ${ }^{2}$ Harvard-Smithsonian Center for Astrophysics, 60 Garden Street, Cambridge, MA 02138, USA \\ Received 2008 October 28; accepted 2009 February 25; published 2009 April 24
}

\begin{abstract}
We present calculations of the heliospheric solar wind charge-exchange (SWCX) emission spectra and the resulting contributions of this diffuse background in the ROSAT $1 / 4 \mathrm{keV}$ bands. We compare our results with the soft $\mathrm{X}$-ray background (SXRB) emission detected in front of 378 identified shadowing regions during the ROSAT AllSky Survey. This foreground component is principally attributed to the hot gas of the so-called Local Bubble (LB), an irregularly shaped cavity of $\sim 50-150 \mathrm{pc}$ around the Sun, which is supposed to contain $\sim 10^{6} \mathrm{~K}$ plasma. Our results suggest that the SWCX emission from the heliosphere is bright enough to account for most of the foreground emission toward the majority of low galactic latitude directions, where the LB is the least extended. On the other hand, in a large part of directions with galactic latitude above $30^{\circ}$, the heliospheric SWCX intensity is significantly smaller than the measured one. However, the SWCX $R 2 / R 1$ band ratio differs slightly from the data in the galactic center direction, and more significantly in the galactic anticentre direction where the observed ratio is the smallest. Assuming that both SWCX and hot gas emission are present and their relative contributions vary with direction, we tested a series of thermal plasma spectra for temperatures ranging from $10^{5}$ to $10^{6.5} \mathrm{~K}$ and searched for a combination of SWCX spectra and thermal emission matching the observed intensities and band ratios, while simultaneously being compatible with O vi emission measurements. In the frame of collisional equilibrium models and for solar abundances, the range we derive for hot gas temperature and emission measure cannot reproduce the Wisconsin $C / B$ band ratio. This implies that accounting for SWCX contamination does not remove these known disagreements between data and classical hot gas models. We emphasize the need for additional atomic data, describing consistently EUV and X-ray photon spectra of the charge-exchange emission of heavier solar wind ions.
\end{abstract}

Key words: interplanetary medium - ISM: bubbles - ISM: general - supernova remnants - X-rays: diffuse background - X-rays: general - X-rays: ISM

Online-only material: color figure

\section{INTRODUCTION}

The diffuse soft X-ray background (SXRB), first observed in the 1970s (Bowyer et al. 1968; Williamson et al. 1974; Sanders et al. 1977) has since been shown to be the sum of local and distant sources. Above $2 \mathrm{keV}$, it is dominated by the extragalactic background, itself a combination of unresolved point sources and warm-hot interstellar medium (WHIM) diffuse emission (Hasinger et al. 1993). At lower energies, it is dominated by the galactic halo (Burrows \& Mendenhall 1991; Snowden et al. 1994), and finally below $0.3 \mathrm{keV}$ it is mainly due to the unabsorbed emission from hot gas filling, the so-called Local Bubble (LB; McCammon et al. 1983; Bloch et al. 1986; Snowden et al. 1990a, 1990b), a cavity devoid of dense gas extended at high latitudes and connected to the halo (Frisch \& York 1983; Welsh et al. 1998; Lallement et al. 2003). The main tools used to disentangle local and distant emission are the "shadowing" experiments, i.e., spatial variations of intensity and spectral characteristics around and toward dense, soft X-ray absorbing clouds (e.g., Herbstmeier et al. 1995). Snowden et al. $(1998,2000)$ used more than 370 ROSAT shadows to produce almost full-sky mapping of the "unabsorbed" component of the emission, i.e., the LB contribution.

This was the generally accepted scenario until the discovery of X-ray emission in comets (Lisse et al. 1996) and the identification of the emission mechanism as charge-exchange (CX) reactions between the highly charged heavy solar wind
(SW) ions and the cometary neutrals (Cravens 1997). Cox (1998) suggested that the CX reactions should also occur between heavy SW ions and interstellar neutrals $(\mathrm{H}$ and $\mathrm{He})$ in interplanetary space and that the resulting X-ray emission (solar wind charge exchange; SWCX) should have an impact on the SXRB interpretation. Cravens (2000) estimated that the quiescent level of SWCX emission could be of the same order as the SXRB component attributed to the LB.

This interplanetary, heliospheric emission is time-dependent because of the intrinsic variable nature of the SW. Short timescale variations tend to be washed out by integration along the line of sight (LOS) and the size of the emitting region (Cravens et al. 2001), but longer term variations, including those related to the solar cycle, can cause more persistent changes in the heliospheric emission level. In addition, there is a contribution from the Earth's magnetosphere, due to charge transfer with exospheric neutrals. Such emission, studied in detail by Robertson et al. (2006), reacts instantaneously to SW variations and magnetosphere shape variations, leading to a high variability and the occurrence of high intensity peaks following solar events. The spectral characteristics of some spectacular enhancements have been recently recorded by the XMM-Newton and Suzaku satellites (Snowden et al. 2004; Henley \& Shelton 2008). For such events both magnetospheric and heliospheric contributions may be present.

Very likely most of the sharp increases of terrestrial origin have been removed from the ROSAT map along with the 
cleaning procedure of the Long Term Enhancements (LTEs Snowden et al. 1994), as well as some heliospheric increases, especially toward the downwind (DW) side of the interstellar flow where the gravitational cone of focused helium is the most reactive region. Indeed, most points on the sky in the ROSAT map were observed several times over the course of at least two days, allowing identification and removal of periods of enhanced emission. A debate is still maintained, though, about the actual level of the quasi-stationary heliospheric contribution to the ROSAT maps of unabsorbed emission. This contribution is extremely difficult to detect from time variations. On the other hand, SCWX and hot gas thermal emission have different spectral properties, i.e., the observed spectral information should help to disentangle the two processes. This is the subject of the present study. For a recent review of all types of SWCX phenomena see Bhardwaj et al. (2007).

The first estimates of the stationary heliospheric contribution (Cravens 2000; Lallement 2004) were based on simplifying assumptions about the spectral characteristics of the SWCX emission. Since then the existence of the SWCX phenomenon has motivated theoretical work on exact photon yield values for the charge-transfer collisions (Kharchenko \& Dalgarno 2000; Pepino et al. 2004), as well as a number of laboratory experiments devoted to the CX emission mechanism. For a recent review see Wargelin et al. (2008).

The spatial distribution of magnetospheric and heliospheric SWCX emission was modeled by Robertson \& Cravens (2003), revealing significant variations in brightness as a function of Earth location, LOS direction, and activity phase. Koutroumpa et al. (2006) computed similar maps for a few specific energy bands after including Pepino et al.'s (2004) detailed CX emission spectra for C, N, O, and Ne ions. Lallement (2004), taking into account the specific viewing geometry of ROSAT showed that the heliospheric background in the $1 / 4 \mathrm{keV}$ band was nearly isotropic and could have been unnoticed in the All-Sky Survey maps, while accounting for a large portion of the signal, and possibly the major part at low galactic latitudes.

Using both the stationary and time-dependent models, Koutroumpa et al. $(2007,2009)$ modeled four high-latitude shadowing observations and showed that in the $3 / 4 \mathrm{keV}$ band, where the oxygen lines ( $\mathrm{O}$ VII triplet at $0.57 \mathrm{keV}$ and $\mathrm{O}$ VIII line at $0.65 \mathrm{keV}$ ) are dominant, the SWCX emission from the heliosphere can account for all the unabsorbed, local component of the SXRB, with no need of an LB emission. In parallel, the SW contribution to the background and its variability have been shown to be responsible for some discrepant measurements (Smith et al. 2007) and for supposedly low-energy counterparts of distant objects (Bregman \& Lloyd-Davies 2006).

A $10^{6} \mathrm{~K}$ plasma, however, has very little emission in the $3 / 4 \mathrm{keV}$ band and mainly emits in the $1 / 4 \mathrm{keV}$ band. The Koutroumpa et al. (2007) results, while not requiring any LB emission, therefore do not preclude the existence of $10^{6} \mathrm{~K}$ gas. Exact calculations of the SWCX spectra and intensities below $0.3 \mathrm{keV}$ are mandatory if one wants to disentangle LB hot gas diffuse emission from the SWCX background. In this paper, we examine the SWCX contribution to the $1 / 4 \mathrm{keV}$ spectral region, compare this contribution to observations and also to contributions from hot gas at different temperatures.

Independently of the SWCX contribution, a number of results have somewhat contradicted the interpretation of the unabsorbed SXRB as the LB $10^{6} \mathrm{~K}$ gas emission.

1. Data from the NASA Extreme Ultraviolet Explorer (EUVE) satellite and from the dedicated CHIPS mission did not detect the EUV emission expected from surrounding $10^{6} \mathrm{~K}$ gas (Jelinsky et al. 1995; Hurwitz et al. 2005). It has been suggested that a very low metal abundance may be responsible for this nondetection, but the required depletion level corresponds to the physical state of very dense clouds, which is unlikely for $10^{6} \mathrm{~K}$, tenuous gas.

2. The pressure of this hot gas derived from the X-ray background is far above the pressure within the local interstellar cloud and other clouds embedded in the LB (Lallement 1998; Jenkins 2002).

3. Low-latitude absorption measurements of highly charged ions such as $\mathrm{Si}$ IV, $\mathrm{C}$ IV, and $\mathrm{O}$ VI formed in conductive interfaces between the hot $\left(10^{6} \mathrm{~K}\right)$ gas and embedded cold:warm clouds do not seem to correspond to expectations from the models (Slavin \& Frisch 2002; Indebetouw \& Shull 2004). Column densities of Si IV and C IV are too small and line widths too narrow (Welsh \& Lallement 2005), and O VI is detected only at the periphery of the local cavity, while one would also expect interfaces between the hot gas and the local clouds (Welsh \& Lallement 2008).

4. Fundamental discrepancies arise also when comparing the Wisconsin sounding rocket survey data in the $B$ and $C$ bands, and the ROSAT All-Sky Survey data in the $R 1$ and $R 2$ bands. The four bands are pictured in Figure 1 (upper panel). In the low energy $(0.1-0.2 \mathrm{keV}) B$ band (Bloch et al. 1986; Snowden et al. 1994), the intensity seems to be higher than what is predicted by thermal emission models. This has been particularly well demonstrated by Bellm \& Vaillancourt (2005) who have made a global study over the $0.1-0.3 \mathrm{keV}$ interval. According to this work, a best fit to all energy bands is provided by very low metallicity gas at $10^{5.85} \mathrm{~K}$, but inspection of their results (see their Figure 4) reveals significant discrepancies between measured and observed ratios for this best-fit solution. Especially, the $B / R 12$ band ratio favors a low temperature $\simeq 10^{5.8} \mathrm{~K}$ (the $B$ band intensity is high and favors a shift of the spectrum toward low energies), while the $R 2 / R 1$ ratio favors temperatures above $10^{6} \mathrm{~K}(R 2$ is relatively high, favoring a shift toward high energies).

Whether or not the existence of the SWCX background can help to explain part or all these contradictions is a question that has now to be addressed. This work is a first step in this direction. In Section 2, we describe the SWCX emission and spectral model we have developed and how we make use in our analysis of the Raymond and Smith (R-S) hot plasma model. In Section 3, we compute the expected SWCX emission and the contribution in ROSAT $R 1$ and $R 2$ bands for each of the 378 shadow regions observed by Snowden et al. (2000). We compare the SWCX $R 1+R 2$ intensity with the unabsorbed component derived by the Snowden et al. (2000) shadow analysis and discuss the distribution of the discrepancies between data and the SWCX model. In Section 4, we compute the SWCX model $R 2 / R 1$ and $B / C$ band ratios, as well as the corresponding ratios for hot gas (R-S model) in collisional equilibrium within a large temperature range. We compare the modeled ratios with the observed band ratios during the two (Wisconsin and ROSAT) surveys. In Section 5, we search for a combination of SWCX and hot gas emission compatible with the observed intensities and band ratios and we compare those solutions with observational constraints from O VI and EUV background measurements. In Section 6, we discuss the results and draw some conclusions. 


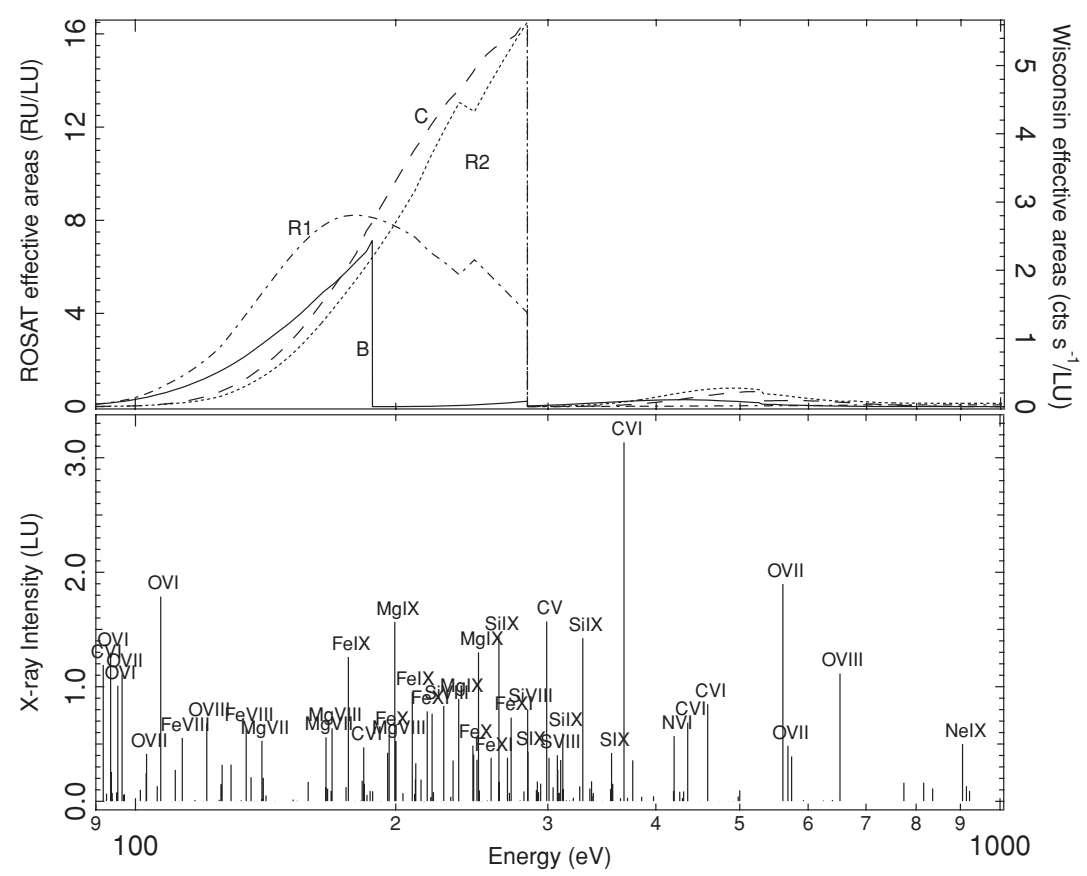

Figure 1. Top panel: Wisconsin $B$ (plain) and $C$ (dashed), and ROSAT $R 1$ (dash-dotted) and $R 2$ (dotted) band effective areas. Bottom panel: example of calculated SWCX spectra in line units (photons $\mathrm{cm}^{-2} \mathrm{~s}^{-1} \mathrm{sr}^{-1}$ ). The emitting ions are marked above the most prominent lines.

\section{MODEL DESCRIPTION}

\subsection{SWCX Model}

The basic model calculating the SWCX emission in the inner heliosphere was thoroughly presented in Koutroumpa et al. (2006); parameters appropriate for the ROSAT All-Sky Survey are discussed in Section 3. We calculate self-consistently the neutral $\mathrm{H}$ and $\mathrm{He}$ density distributions in the inner heliosphere (up to $\sim 100 \mathrm{AU}$ ), in response to solar gravity, radiation pressure, and anisotropic ionization processes for the two neutral species. Ionization of $\mathrm{H}$ atoms is mainly due to their $\mathrm{CX}$ collisions with SW protons and He atoms are mostly ionized by solar EUV photons and electron impact. We also consider the impact of $\mathrm{CX}$ on the SW ion distributions. This interaction is described in the following reaction:

$$
\mathrm{X}^{Q+}+[\mathrm{H}, \mathrm{He}] \rightarrow \mathrm{X}^{*(Q-1)+}+\left[\mathrm{H}^{+}, \mathrm{He}^{+}\right] .
$$

The collision rate per volume unit $R_{\mathrm{X}} Q^{++}\left(\mathrm{cm}^{-3} \mathrm{~s}^{-1}\right)$ of $\mathrm{X}^{Q+}$ ions with the neutral heliospheric atoms is given by the equation:

$$
\begin{aligned}
R_{\mathrm{X} \varrho^{++}}(r) & =N_{\mathrm{X}^{\varrho^{+}}}(r) v_{r}\left(\sigma_{\left(\mathrm{H}, \mathrm{X}^{{ }^{+}}\right)} n_{\mathrm{H}}(r)+\sigma_{\left(\mathrm{He}, \mathrm{X}^{{ }^{+}}\right)} n_{\mathrm{He}}(r)\right) \\
& =R_{\left(\mathrm{X}^{\varrho^{+}}, \mathrm{H}\right)}(r)+R_{\left(\mathrm{X} \varrho^{+}, \mathrm{He}\right)}(r),
\end{aligned}
$$

where $\sigma_{\left(\mathrm{H}^{\mathrm{X}}{ }^{\left.Q^{+}\right)}\right.}$and $\sigma_{\left(\mathrm{He}, \mathrm{X}^{Q+}\right)}$ are the hydrogen and helium CX cross sections, $n_{\mathrm{H}}(r)$ and $n_{\mathrm{He}}(r)$ are the hydrogen and helium density distributions, respectively, $\bar{v}_{r}=\bar{V}_{\mathrm{SW}}-\bar{v}_{n} \approx \bar{V}_{\mathrm{SW}}$ the relative velocity between SW ions and interstellar (IS) neutrals in the inner heliosphere, and $N_{\mathrm{X}^{+}}(r)$ is the self-consistent solution to the differential equation:

$$
\begin{aligned}
& \frac{d N_{\mathrm{X}^{\ell^{+}}}}{d x}=-N_{\mathrm{X}^{\ell^{+}}}\left(\sigma_{\left(\mathrm{H}, \mathrm{X}^{\ell+}\right)} n_{\mathrm{H}}(x)+\sigma_{\left(\mathrm{He}, \mathrm{X}^{\varrho+}\right)} n_{\mathrm{He}}(x)\right) \\
& +N_{\mathrm{X}^{(Q+1)+}}\left(\sigma_{\left(\mathrm{H}, \mathrm{X}^{(Q+1)+}\right)} n_{\mathrm{H}}(x)+\sigma_{\left(\mathrm{He}, \mathrm{X}^{(Q+1)+}\right)} n_{\mathrm{He}}(x)\right)
\end{aligned}
$$

expressing the evolution of the density distribution of ion $\mathrm{X}^{Q+}$ along SW streamlines due to production (from CX reactions of ion $\mathrm{X}^{(Q+1)+}$ ) and loss terms.
Cross section uncertainties are mainly due to instrumental systematic errors and most important to the collision energy dependence of cross sections. Detailed uncertainties for individual ions are not given in literature, but average uncertainties of $\sim 30 \%$ at most are reported (Wargelin et al. 2008).

Then, we establish emissivity grids in units of (photons $\left.\mathrm{cm}^{-3} \mathrm{~s}^{-1}\right)$ :

$$
\varepsilon_{i}(r)=R_{\left(\mathrm{X}^{\left.\ell^{+}, \mathrm{H}\right)}\right.}(r) Y_{\left(E_{i}, \mathrm{H}\right)}+R_{\left(\mathrm{X}^{\left.Q^{+}, \mathrm{He}\right)}\right)}(r) Y_{\left(E_{i}, \mathrm{He}\right)},
$$

where $Y_{\left(E_{i}, \mathrm{M}\right)}$ is the photon emission yield (in number of photons) computed for a spectral line of photon energy $E_{i}$ following $\mathrm{CX}$ with the corresponding neutral species $\mathrm{M}(\mathrm{H}$ or $\mathrm{He}$ individually). For any LOS and observation date, the directional intensity of this spectral line is given by

$$
I_{E_{i}}(L U)=\frac{1}{4 \pi} \int_{0}^{\sim 100 \mathrm{AU}} \varepsilon_{i}(s) d s,
$$

which defines the average intensity, in line units ( $\mathrm{LU}=$ photons $\mathrm{cm}^{-2} \mathrm{~s}^{-1} \mathrm{sr}^{-1}$ ), of the spectral line for the particular date and LOS, as well as the solar cycle phase (minimum or maximum) corresponding at this date. The intensity is somewhat underestimated because of the SW ion propagation in the heliosheath up to the heliopause, and in the heliotail up to $\sim 3000 \mathrm{AU}$, where all ions are used up. The outer heliospheric region is neglected in our model, but estimates yield a maximum additional $\sim 20 \%$ contribution in the DW direction, with possible effects on the SWCX spectral hardness (see Section 4).

Our original atomic database (Kharchenko 2005) included $\mathrm{C}^{5,6+}, \mathrm{N}^{5,6,7+}, \mathrm{O}^{6,7,8+}, \mathrm{Ne}^{8,9+}$, and $\mathrm{Mg}^{10,11+}$ ions. Exact calculations of the cascading photon spectra were performed individually for these ions when they CX with hydrogen and helium, respectively. Detailed CX collision cross sections taking into account both the neutral target species and the SW velocity regime were include in the calculations (P. Stancil 2003, private communication). These calculations have already been used to 
reproduce observed SWCX spectra from comets with CHIPS (Sasseen et al. 2006).

The database was recently updated to include $\mathrm{Fe}^{7 \ldots 13+}$, $\mathrm{Si}^{5 \ldots 10+}, \mathrm{S}^{6 \ldots 11+}$, and $\mathrm{Mg}^{4 \ldots 9+}$ ions that emit intense lines in the $0.1-0.3 \mathrm{keV}$ range. Individual emission spectra induced in the CX collisions of these ions have very complicated structures because of a large number of intermediate multiplets related to different excited states of many-electron ions. The photon yields $Y(E, M)$ for heavier ions were calculated using the simplified hydrogenic model (Kharchenko \& Dalgarno 2000), which assumes a hydrogenic nature of electronic excited states. In this model, the effective charge of hydrogenic ion is computed from an accurate value of the ion ionization potential and branching ratios of radiative cascading transitions are chosen to be the same as in all $\mathrm{H}$-like ions. Moreover, photon yields were calculated using a single neutral species, which means that no distinction between $\mathrm{H}$ and $\mathrm{He}$ was made. The hydrogenic approximation of the CX emission spectra is a quantum mechanical model in which an actual ion spectra may be replaced with the hydrogenic spectra. In this model, the total energy of emitted photons is defined by an initial state population and should be an accurate quantity matching real spectra. Positions of emission lines do not correspond exactly to real emission spectra, but this defect is not very important at the low resolution of the observed spectra. Total cross sections of CX collisions for the hydrogenic approximations have been calculated using the overbarrier model (Kharchenko \& Dalgarno 2001). An example of calculated spectra is presented in Figure 1(lower panel), with the emitting ion identifying the most intense lines.

\subsection{Hot Gas Thermal Emission}

We use an R-S hot plasma model (Raymond \& Smith 1977) assuming typical metal abundances [He, C, N, O, Ne, Mg, Si, S, $\mathrm{Ar}, \mathrm{Ca}, \mathrm{Fe}, \mathrm{Ni}]=[10.93,8.52,7.96,8.82,7.96,7.52,7.60,7.20$, $6.90,6.30,7.60,6.30]$ (Allen 1973). We use this model rather than the APEC model (Smith et al. 2001) that superseded it because we are most interested in the $1 / 4 \mathrm{keV}$ range. APEC includes only transitions for which accurate atomic rates are available, while the code of R-S estimates the emission in the large number of weak lines that are known to be present (e.g., from moderately ionized species of $\mathrm{Mg}, \mathrm{Si}, \mathrm{S}$, and $\mathrm{Fe}$ ) but which lack accurate excitation rates and wavelengths. Given the low spectral resolution of the observations considered here and our interest in the total emitted power, the R-S model serves very well.

This model gives us X-ray emissivities $f_{1}(T)$ and $f_{2}(T)$ convolved by and summed in the ROSAT $R 1$ and $R 2$ bands, respectively, as a function of temperature such that the total hot gas X-ray flux in these bands is defined as

$$
I_{12, \mathrm{LB}}=I_{1, \mathrm{LB}}+I_{2, \mathrm{LB}}=\operatorname{EM}(i, T) \cdot\left(f_{1}(T)+f_{2}(T)\right),
$$

where $\operatorname{EM}(i, T)$ is the emission measure for temperature $T$ and look direction $i$. Units of functions $f_{1}$ and $f_{2}$ are $\mathrm{RUEM}^{-1}$, where $\mathrm{RU}=10^{-6}$ counts $\mathrm{s}^{-1} \operatorname{arcmin}^{-2}$ is the usual ROSAT detector unit and EM is the typical emission measure unit $\mathrm{cm}^{-6} \mathrm{pc}$.

Equivalently, the hot gas emission in bands $B$ and $C$ is defined as

$$
I_{B C, \mathrm{LB}}=I_{B, \mathrm{LB}}+I_{C, \mathrm{LB}}=\operatorname{EM}(i, T) \cdot\left(f_{B}(T)+f_{C}(T)\right),
$$

where $f_{B}(T)$ and $f_{C}(T)$ are the equivalent emissivity functions in the $B$ and $C$ bands derived by the R-S plasma code, in units of counts s ${ }^{-1} \mathrm{EM}^{-1}$.

\section{3. $R 1+R 2$ INTENSITIES}

We have calculated SWCX spectra in ROSAT observation geometry for the shadow field LOS listed in Table 1 of Snowden et al. (2000), which were observed during the ROSAT AllSky Survey. The shadows analyzed by Snowden et al. (2000) were located at high galactic latitudes and in general above $15^{\circ}$ from the galactic plane. The ROSAT observation geometry is defined with the view direction perpendicular to the Sunsatellite direction. Thus, it takes a six-month period to build a full-sky map of the soft X-ray intensity. The ROSAT All-Sky Survey was performed between 1990 July and 1991 February, which corresponds to maximum solar activity conditions that were taken into account in the SWCX simulations.

Maximum solar activity conditions imply the following input parameters in the SWCX model. We consider a radiation pressure to gravity ratio $\mu=1.5$ for neutral hydrogen and slightly anisotropic ionization rates varying between $8.4 \times$ $10^{-7} \mathrm{~s}^{-1}$ at the solar equator and $6.7 \times 10^{-7} \mathrm{~s}^{-1}$ at the poles (Quémerais et al. 2006). For neutral helium, the average lifetime (inverse ionization rate) at $1 \mathrm{AU}$ is $0.62 \times 10^{7} \mathrm{~s}$ at solar maximum, in agreement with McMullin et al. (2004). In solar maximum, the SW is considered to be a complex mix of slow and fast wind states, that is, in general approximated with an average slow wind flux. Slow SW flows at $\sim 400 \mathrm{~km} \mathrm{~s}^{-1}$ and has a proton density of $\sim 6.5 \mathrm{~cm}^{-3}$ at the Earth position. The oxygen content with respect to protons is $[\mathrm{O} / \mathrm{H}]=1 / 1780$. The most important heavy ion charge state abundances with respect to oxygen $\left[\mathrm{X}^{q+} / \mathrm{O}\right]$ are $\mathrm{C}^{5,6+}:[0.21,0.318], \mathrm{O}^{6,7,8+}:[0.73$, $0.2,0.07], \mathrm{Si}^{8,9,10+}:[0.057,0.049,0.021]$, and $\mathrm{Fe}^{8,9,10,11+}:[0.034$, 0.041, 0.031, 0.023] (adopted from Schwadron \& Cravens 2000).

We have convolved the individual spectra with the ROSAT $R 1$ and $R 2$ band responses in order to calculate the total SWCX flux in these bands, as well as the total $R 12(R 1+R 2)$ flux. We plot the resulting $R 12$ SWCX flux and the unabsorbed $I_{12 \text {,obs }}$ component from the Snowden et al. (2000) analysis as a function of absolute galactic latitude in Figure $2 . I_{12 \text {,obs }}$ corresponds to the unabsorbed portion of the SXRB that was originally attributed to the $\mathrm{LB} \sim 10^{6} \mathrm{~K}$ hot gas. $\mathrm{X}$-ray intensities are presented in ROSAT units ( $\mathrm{RU}=10^{-6}$ counts $\mathrm{s}^{-1} \operatorname{arcmin}^{-2}$ ).

The SWCX R12 flux (black dots) varies between 212 and 460 RU with an average value of 332 RU and is fairly uniformly distributed across all latitudes. The lower and upper limits calculated in the SWCX simulations for average maximum conditions are represented with the plain black horizontal lines.

On the other hand, the unabsorbed $I_{12 \text {,obs }}$ component (gray circles) derived in the Snowden et al. (2000) analysis has a clear correlation with the absolute galactic latitude. Higher $I_{12, \text { obs }}$ values are measured toward higher latitudes, where the local cavity is enlarged and communicates with the galactic halo through the chimneys.

In the figure, it is clear that the SWCX intensity is of the same order as the $I_{12 \text {,obs }}$ intensity measured in low galactic latitudes (up to around $20^{\circ}-25^{\circ}$ ). We can conclude, then, that the SWCX $1 / 4 \mathrm{keV}$ flux could account for most of the observed ROSAT emission in the galactic plane. This conclusion implicitly assumes that the highly peaked exospheric SWCX contribution has been cleaned from the ROSAT data, but not the heliospheric contribution.

Figure 3 is a map in galactic coordinates of the ratio between the unabsorbed ROSAT emission and the computed SWCX contribution. The map clearly reveals the emission from the so-called chimneys that connect the local cavity to the northern 


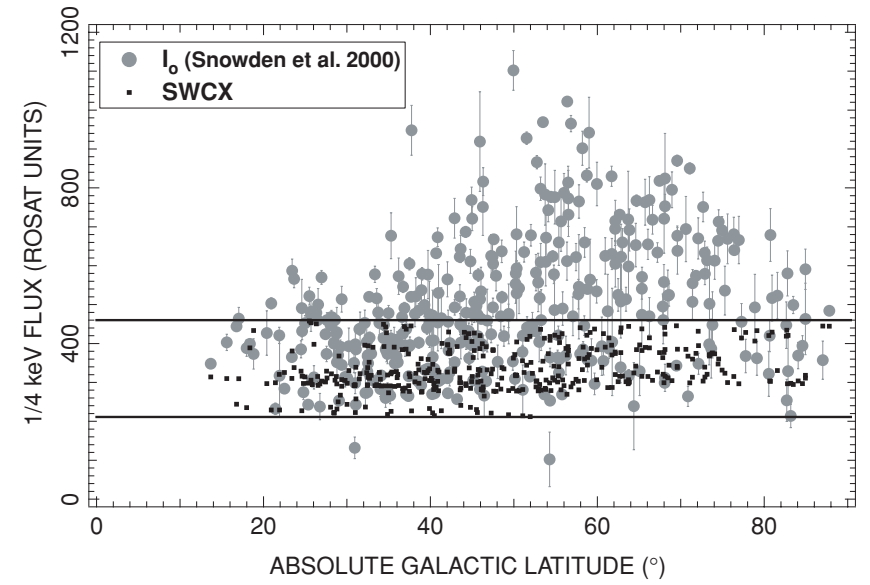

Figure 2. Measured unabsorbed $\left(I_{12, \mathrm{obs}}\right.$, from Snowden et al. (2000), gray circles) and calculated SWCX (black dots) fluxes in the ROSAT $1 / 4 \mathrm{keV}$ $(R 1+R 2)$ band as a function of absolute galactic latitude for 378 shadowing observations. Flux is given in ROSAT Units $\left(10^{-6}\right.$ counts s${ }^{-1}$ arcmin $\left.^{-2}\right)$.

and the southern halo. Our intensity results do not preclude that outside these chimneys the totality of the signal is SWCX emission.

\section{BAND RATIOS}

For each SWCX spectrum calculated in the look directions presented in Figure 2, we have calculated the $R 2 / R 1$ (ROSAT) and $C / B^{3}$ (Wisconsin) ratios. We find an average $R 2 / R 1$ (hereafter $R_{\mathrm{CX}}$ ) ratio of 1.39 and an average $C / B$ ratio of 6.67 . Although both ratios show very little variation across the sky, there is a hardness trend of the SWCX spectra with harder spectra toward the DW direction (UW to DW variations: $R_{\mathrm{CX}}=$ [1.36-1.41], $C / B=[6.25-7.14])$. However, we need to alert the reader that these are somewhat uncertain SWCX spectra and therefore somewhat uncertain band ratios. Indeed, as we mentioned in Section 2, exact calculations for $\mathrm{Fe}, \mathrm{Si}, \mathrm{Mg}$, and $\mathrm{Al}$

\footnotetext{
3 The original papers on the Wisconsin survey referred to the $B / C$ ratio, but given the extensive use of the $R 2 / R 1$ ratio in our analysis and the rough correspondence between $R 1$ and $B$, and $R 2$ and $C$, we refer to the $C / B$ ratio.
}

are not yet available, and no distinction was done between the neutral targets $(\mathrm{H}$ or $\mathrm{He})$, while laboratory experiments show that the energy levels populated after the electron capture and the subsequent radiative cascades may differ significantly for different targets. Since most of the SWCX DW emission is due to the interaction with neutral helium, while on the upwind (UW) side hydrogen is the main contributor, more precise calculations could have an effect on the hardness. Also, although preliminary calculations show an almost negligible effect, the heliospheric model cutoff (especially in the DW directions) may be responsible for the "loss" of relatively more emission from lower charge states (at relatively lower energies) than emission from higher charge states (at relatively higher energies). Thus, the calculated SWCX spectra may actually be softer than what is predicted here. It is evident that a more detailed calculation taking into account all metals and the neutral target nature, as well as detailed cascading collisions (secondary ion production) in the outer heliosphere is needed in the future. On the other hand, the interval we find for the ratio can be used a reliable value for the average SWCX ratio.

These SWCX ratios have to be compared with the corresponding ratios for thermal emission. The latter were obtained as a function of temperature by convolving the R-S spectra with the ROSAT band responses $R 1$ and $R 2$ and Wisconsin $B$ and $C$ responses. The results are shown in Figure 4. Above $\log T=6.1$ the thermal $R 2 / R 1$ ratio reaches its maximal value of $\simeq 1.2$. It remains, however, slightly lower than the SWCX ratio of 1.361.41. At those temperatures, the thermal $C / B$ ratio increases to its maximal value of $\simeq 4$, a value almost half the SWCX ratio of 6.24-7.14, i.e., a significant difference. Those curves allow estimates of the ratios for combinations of thermal plus SWCX background emissions.

\section{COMBINATION OF THE HELIOSPHERIC SWCX AND LB HOT PLASMA EMISSION}

For a comparison with the data we consider two regions: one centered on the direction of the incoming IS flow at ecliptic coordinates $(\lambda, \beta) \sim(252.3,8.5)^{\circ}$ for the IS $\mathrm{H}$ flow, according to Lallement et al. (2005) (UW direction) and one looking at the

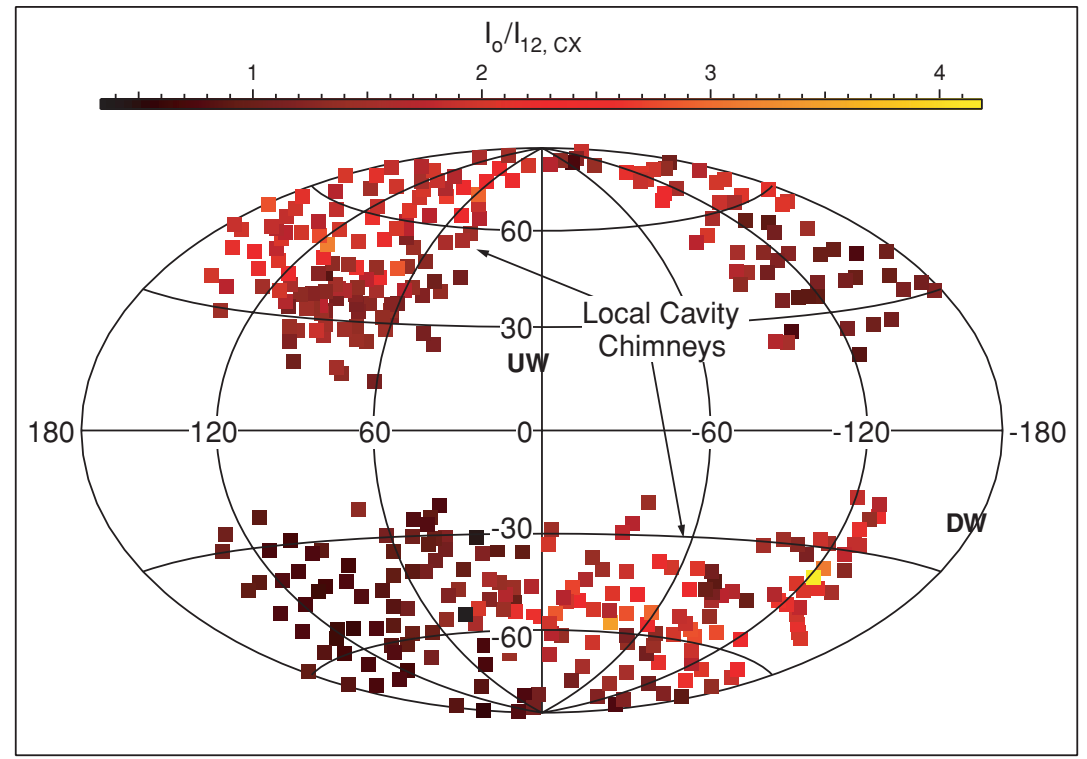

Figure 3. Unabsorbed $I_{12 \text {,obs }} R 12$ flux data over simulated $I_{12, \mathrm{CX}}$ SWCX flux ratio map in galactic coordinates with an equal-area Aitoff projection. For information, we note the UW and DW directions in the sky. The Local Cavity (LC) chimneys appear clearly where the $I_{12, \text { obs }} / I_{12, C X}$ ratio is the highest.

(A color version of this figure is available in the online journal.) 
Table 1

Temperature $(\log T)$ Limits and EM Solutions for Upwind (UW) and Downwind (DW) Look Directions When Combining an SWCX and R-S Hot Plasma Code

\begin{tabular}{|c|c|c|c|c|c|c|c|c|c|c|}
\hline \multirow{2}{*}{$\begin{array}{l}\text { Look } \\
\text { Direction }\end{array}$} & \multicolumn{5}{|c|}{ Local Bubble } & \multicolumn{3}{|c|}{ SWCX } & \multicolumn{2}{|c|}{$(\mathrm{LB}+\mathrm{SWCX})^{\mathrm{c}}$} \\
\hline & $\log T$ & $\begin{array}{c}\text { E M } \\
\left(10^{-4} \mathrm{~cm}^{-6} \mathrm{pc}\right)\end{array}$ & $\begin{array}{r}I_{12, \mathrm{LB}} \\
(\mathrm{RU})\end{array}$ & \multicolumn{2}{|c|}{$\left(\right.$ counts s $\left.^{-1}\right)$} & $\begin{array}{c}I_{12, \mathrm{CX}}{ }^{\mathrm{b}} \\
(\mathrm{RU})\end{array}$ & \multicolumn{2}{|c|}{$\left(\right.$ counts s $\left.^{-1}\right)$} & $\begin{array}{c}I_{B C} \\
\left(\text { counts s }^{-1}\right)\end{array}$ & $C / B$ \\
\hline UW & 5.64 & 5.5 & 25 & 3.8 & 2.6 & 300 & 10. & 66.5 & 82.9 & 5. \\
\hline DW & 21. & 96 & 14.5 & 10. & 354 & 11.8 & 78.8 & 115.1 & 3.37 & \\
\hline UW & 6.00 & 3.7 & 62 & 4.8 & 11.1 & 263 & 9.2 & 58. & 82.7 & 5. \\
\hline \multirow[t]{3}{*}{ DW } & & 14.1 & 238 & 18.3 & 42.5 & 212 & 7.7 & 46.5 & 114.1 & 3.57 \\
\hline & \multicolumn{4}{|c|}{ Observational Input } & & & & & & \\
\hline & $I_{12, \mathrm{obs}}(\mathrm{RU})$ & $R 2 / R 1\left(R_{\mathrm{obs}}\right)$ & $I_{B C}\left(\right.$ counts s$\left.{ }^{-1}\right)$ & $C / B$ & & & & & & \\
\hline UW & 325 & 1.25 & $\sim 90$ & 4. & & & & & & \\
\hline DW & 450 & 1.04 & $\sim 125$ & 2.17 & & & & & & \\
\hline
\end{tabular}

Notes. In the lower part of the table, we include the observational input considered.

${ }^{\mathrm{a}} I_{B}=\mathrm{EM} \cdot f_{B}(T), I_{C}=\mathrm{EM} \cdot f_{C}(T)$.

${ }^{\mathrm{b}} I_{12, \mathrm{CX}}=I_{12, \mathrm{obs}}-I_{12, \mathrm{LB}}$.

${ }^{\mathrm{c}} I_{B C}=I_{B}(\mathrm{LB}+\mathrm{CX})+I_{C}(\mathrm{LB}+\mathrm{CX}), C / B=I_{B}(\mathrm{LB}+\mathrm{CX}) / I_{C}(\mathrm{LB}+\mathrm{CX})$

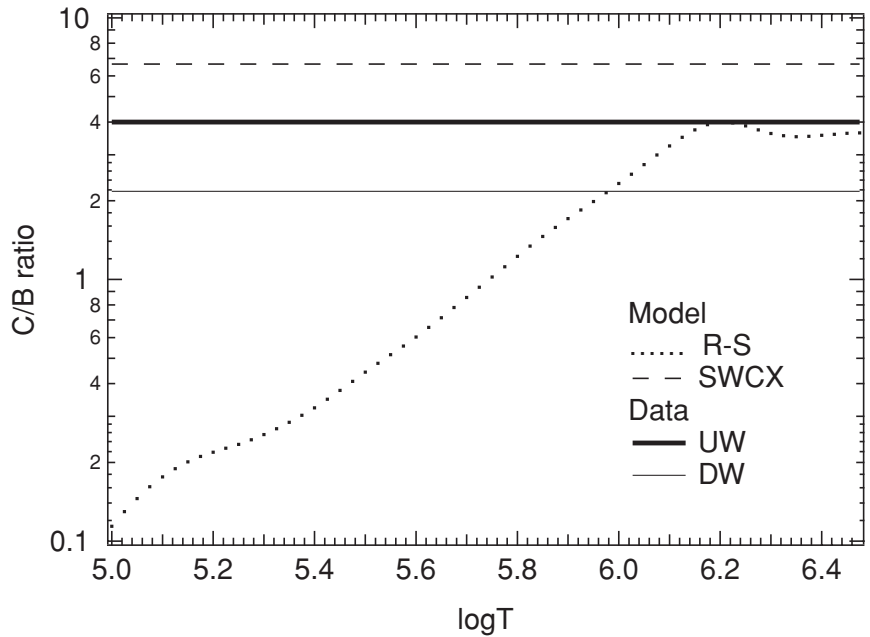

by Snowden et al. (1990b), which define the so-called color gradient axis of the SXRB. For these two regions, we can derive average values of observed unabsorbed $1 / 4 \mathrm{keV}$ emission using the LB contours in the Snowden et al. (1998) analysis. For the UW direction, the ROSAT unabsorbed $I_{12 \text { obs }}$ emission we estimate $\sim 325 \mathrm{RU}$, while for the DW direction the observed unabsorbed level is found to be $450 \mathrm{RU}$.

The equivalent $B+C$ intensities in the Wisconsin survey are estimated on average $\sim 90$ and $\sim 125$ counts s$^{-1}$ for the UW and DW directions, respectively (Snowden et al. 1990b). However, those intensities include both the foreground (assumed LB) and more distant components (galactic halo and extragalactic), since the Wisconsin survey did not have enough spatial resolution to study the shadowing fields. Moreover, the Wisconsin sounding rocket measurements looked in the roughly anti-Sunward direction, which should also affect the comparison with the ROSAT All-Sky Survey in terms of the SWCX component spatial distribution. For instance, for the DW look directions, the Wisconsin sounding rockets were observing directly through the He cone and should have had a higher "contamination" of SWCX emission than the ROSAT detectors that must have been located in crosswind positions on the Earth's orbit in order to observe in the DW directions.

The measured $R 2 / R 1$ and $C / B$ ratios for these regions are shown superimposed to the models in Figure 4 and listed in Table 1 . For the UW area, the measured $R 2 / R 1$ ratio is close to the SWCX value, although slightly smaller, while for the DW area it is significantly smaller. For both areas, the measured $C / B$ ratio is lower than the SWCX ratio. Figure 4 shows that for both areas, a combination of SWCX and thermal emissions may, in principle, account for the ROSAT measurements, the thermal emission lowering the $R 2 / R 1$ ratio to achieve the observed value. Similarly, independently of the $R 2 / R 1$ ratio, Figure 4 also shows that a combination of both backgrounds may account for the Wisconsin data, the thermal emission decreasing the $C / B$ ratio to achieve the observed value.

It remains to find a combination satisfying both ratios simultaneously. Our attempt to find a solution is the following one. For each assumed temperature of the hot gas, we use the $R 1$ and $R 2$ data (and thus the observed ratio) and the SWCX spectral shape to derive the respective contributions of SWCX and thermal emission, i.e., we derive which quantity of SWCX induced $R 12$ intensity and which emission measure EM for the hot gas led to the measured intensities and the measured $R 2 / R 1$ ratio.

outgoing flow direction (DW direction). In galactic coordinates the UW direction corresponds to $(l, b)=(5.1,19.6)^{\circ}$, close to the galactic center direction (antigalactic direction for DW, respectively). These two regions are also very close to the minimum and maximum values of the hardness ratio derived 

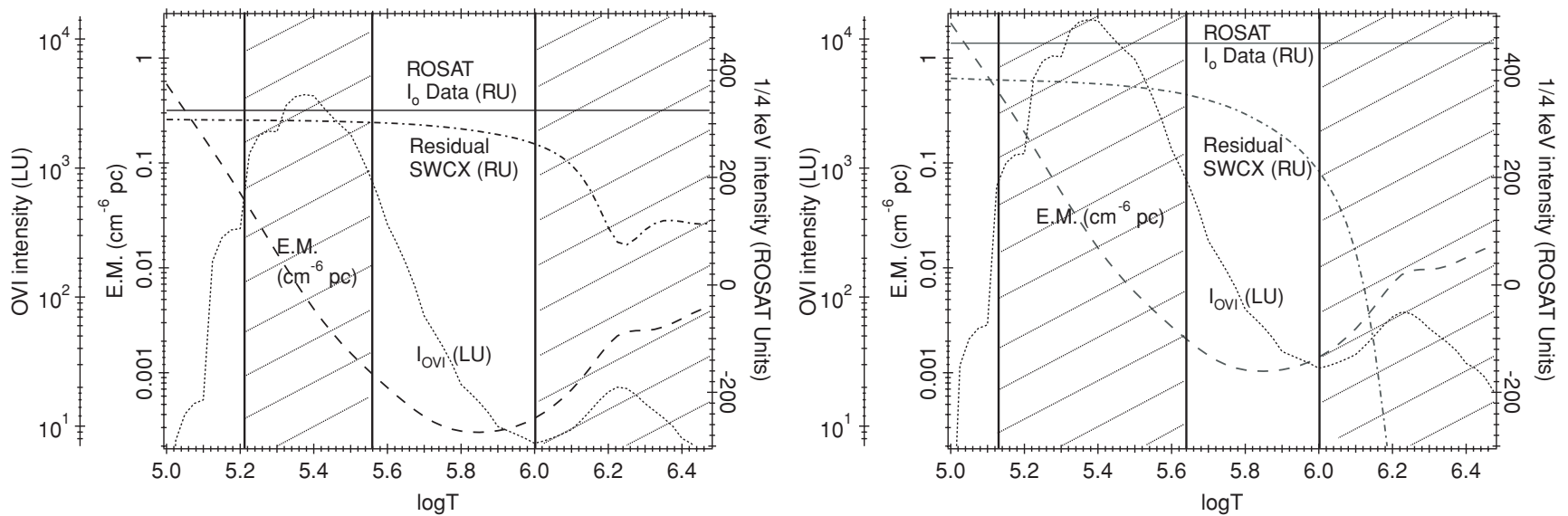

Figure 5. Solutions for the EM (dashed line) and residual SWCX emission (dot-dashed line) as a function of temperature of a hot plasma for observed $R 12$ intensities $\left(I_{12}\right.$,obs, plain horizontal lines) for an upwind (left panel) and downwind (right panel) look directions. Upper O vi doublet intensity limit (dotted line) is calculated for the EM values. Discontinuities in the O VI intensity curve are due to O VI density interpolation in the Chianti database. The cross-hatched regions show the hot gas temperature ranges excluded by O VI intensity (Shelton 2003) and SWCX emission constraints.

We a posteriori calculate the $B$ and $C$ intensities, $C / B$ ratio, and the $\mathrm{O}$ vI emission of the hot gas and compare with the data.

The SWCX model predicts a total $R 12$ intensity:

$$
\begin{aligned}
I_{12, \mathrm{CX}}=I_{1, \mathrm{CX}}+I_{2, \mathrm{CX}} & =I_{1, \mathrm{CX}} \cdot\left(1+R_{\mathrm{CX}}\right) \\
& =I_{2, \mathrm{CX}} \cdot \frac{1+R_{\mathrm{CX}}}{R_{\mathrm{CX}}},
\end{aligned}
$$

where $R_{\mathrm{CX}}$ is the $R 2 / R 1$ ratio predicted by the SWCX model.

The total unabsorbed flux $I_{12, \text { obs }}(i)$ measured in the $R 12$ band toward look direction $i$ is the sum of LB hot gas $I_{12, \mathrm{LB}}$ and SWCX $I_{12, \mathrm{CX}}$ fluxes: $I_{12, \text { obs }}(i)=I_{12, \mathrm{CX}}+I_{12, \mathrm{LB}}$, so that SWCX intensity can be written as

$I_{12, \mathrm{CX}}=I_{12, \mathrm{obs}}(i)-I_{12, \mathrm{LB}}=I_{12, \mathrm{obs}}(i)-\mathrm{EM} \cdot\left(f_{1}(T)+f_{2}(T)\right)$.

The observed $R 2 / R 1$ ratio (hereafter $R_{\mathrm{obs}}$ ) toward look direction $i$ is defined by the equation

$$
R_{\mathrm{obs}}(i)=\frac{I_{2, \mathrm{CX}}+I_{2, \mathrm{LB}}}{I_{1, \mathrm{CX}}+I_{1, \mathrm{LB}}}
$$

Resolving Equation (11) by using Equations (6)-(10), we find the hot gas emission measure $\operatorname{EM}(i, T)$ as a function of temperature, total $R 12$ measured intensity $I_{12 \text {,obs }}(i)$ and measured $R_{\text {obs }}(i)$ ratio toward look direction $i$ :

$\mathrm{EM}(i, T)=\frac{R_{\mathrm{CX}}-R_{\mathrm{obs}}(i)}{\left(R_{\mathrm{obs}}(i)+1\right) \cdot\left(R_{\mathrm{CX}} \cdot f_{1}(T)-f_{2}(T)\right)} \cdot I_{12, \mathrm{obs}}(i)$

We calculate the emission measure for the two UW and DW directions defined above using the following numerical values: (1) the $R_{\mathrm{CX}}$ ratio is constant and equal to 1.39 , (2) observed values of the unabsorbed portion of the $1 / 4 \mathrm{keV}$ emission in the $R 12$ band are $I_{12, \text { obs }}(\mathrm{UW}, \mathrm{DW})=(325,450) \mathrm{RU}$ as derived from the LB contours in the Snowden et al. (1998) analysis for the UW and DW (respectively galactic and antigalactic) directions, and (3) the corresponding observed $R 2 / R 1$ ratio is $R_{\mathrm{obs}}=1.25$ and 1.04 for the UW and DW directions, respectively.

In Figure 5, we show the resulting EM and the portion of the total emission due to the SWCX mechanism (called residual emission) as derived from Equations (12) and (10), respectively, as a function of $\log T$ and for the two look lines. We show superimposed the $R 12$ measured intensities in those directions.
For the calculated EM and corresponding temperatures we have also added to Figure 5 the intensity of the $\mathrm{O}$ VI doublet at $\bar{\lambda}=1034 \AA$ (1032 and $1038 \AA$ ). In order to calculate this $\mathrm{O}$ vi doublet emission, we have used Equation (5) of Shull \& Slavin (1994) and assumed that interstellar O abundance is 8.5 $\times 10^{-4}$. We also assume that the $\mathrm{O}$ VI ion proportion depends on temperature according to the Chianti database formulas for collisional equilibrium (Landi et al. 2006).

In order to delimit the possible temperature solutions for the LB hot gas, we place the following constraints: (1) We assume that the SWCX model is accurate enough to ensure that the heliospheric emission in the $R 12$ band cannot be lower than $\sim 212$ RU (lower limit in Figure 2). This gives us (from the right panel of Figure 5) an upper limit of $\log T=6$ in temperature. (2) We use the observed upper limit of O vi doublet intensity, reported at $\sim 800 \mathrm{LU}$ (Shelton 2003), which gives us two temperature limits at $\log T=5.13$ and 5.64 (extreme limits in the DW direction). The interval between those two temperatures is forbidden because the corresponding O VI column densities (and thus the $\mathrm{O}$ vI intensity) are too high to match observations. Temperatures below $10^{5.13} \mathrm{~K}$ would predict extremely strong $\mathrm{C}$ VI and N VI absorption toward nearby stars, which have not been observed (e.g., Lehner et al. 2003) so we do not consider it a realistic solution. The limits of valid temperature intervals are marked by the vertical bold lines and the cross-hatched regions in Figure 5 show the excluded temperature ranges. The two most plausible hot gas temperature limits $(\log T=5.64,6.0)$ along with the corresponding UW and DW emission measures and residual SWCX emission are summarized in Table 1.

We also calculate for the two boundary solutions the corresponding SWCX intensities and the thermal emission intensities in the $B$ and $C$ bands by convolving our simulated SWCX and the hot gas spectra with the band responses. For the two temperatures, the total hot gas and SWCX intensity in the $B+C$ band $\left(I_{B C}\right.$ in Table 1) is found to be about 83 counts s ${ }^{-1}$ and about 115 counts $\mathrm{s}^{-1}$ for the UW and DW directions, respectively. This similarity arises from the similarity between the wavelength intervals covered by the $B$ and $C$ bands and the $R 1$ and $R 2$ bands (see Figure 1). The $C / B$ ratio derived from this analysis is 5.0 and $\sim 3.5$ for the UW and DW directions accordingly, for temperatures above $10^{5.64} \mathrm{~K}$.

The $(B+C)$ total intensity is consistent with the lower values reported in the Wisconsin survey (Snowden et al. 1990b), which correspond to the lower galactic latitudes. Moreover, Snowden 
et al. (1990b) did not proceed with a shadowing analysis of the Wisconsin data, so the reported values include both local and more distant absorbed components and are expected to be higher than the hot gas and SWCX combination we present here.

However, the $C / B$ ratio computed in the analysis (5 to $\sim 3.6$ from UW to DW, depending on temperature) is inconsistent with the observed value, especially in the DW direction (observed $\sim 2.2$ ), suggesting that we should need more hot gas emitting in the $B$ band. This inconsistency cannot be attributed to the absorbed portion of emission included in the Wisconsin data analysis because the absorbed component is a high- $T$ gas giving a harder spectrum since absorption is more effective in lower energies.

This inconsistency of the DW $C / B$ ratio is important, since it seems difficult to explain in the context of our study. As a matter of fact, as can be seen in Table 1, the SWCX contribution in the $C$ band is large whatever the temperature, and reaching a $C / B$ ratio of 2.2 requires a very small SWCX emission, in our sense far from realistic. Again, as we discussed in the introduction and it was shown in the Bellm \& Vaillancourt (2005) study, the $B$ intensity is higher than expected from the models. This seems to remain true (and even worse) when taking into account the SWCX contribution.

\section{DISCUSSION AND CONCLUSIONS}

We have modeled the intensity and spectral characteristics of the heliospheric SWCX emission at the time of the ROSAT survey and compared with the unabsorbed, local emission derived by Snowden et al. (2000) in the $1 / 4 \mathrm{keV}$ band. The results show that the SWCX emission can account for most of the total intensity recorded in the $R 1+R 2$ bands for most of low-latitude LOS. A map of the heliospheric SWCX portion of the total signal clearly reveals the high-latitude chimneys to the halo as the only regions unambiguously dominated by hot gas emission. Such a result can be interpreted as meaning that little or no hot gas exists within the galactic disk.

The spectral characteristics, however, reveal more complexity and preclude such a simple scenario. The SWCX band ratios disagree with the observations, especially toward the galactic anticenter and at low energies $(C / B)$. We have thus searched for a combination of SWCX and thermal emission from hot gas in equilibrium and solar abundances able to reproduce the data. Our study shows that a combination of SWCX and thermal emission can reproduce the data in the galactic center hemisphere at low latitudes. For this solution, the SWCX emission strongly dominates. The temperature of the hot gas is constrained within the interval $10^{5.64}-10^{6}$. The upper limit is constrained by the lower limit on the SWCX intensity. This upper limit can be considered as firmly determined, thanks to recent observational studies above $0.3 \mathrm{keV}$ that have confirmed the validity of our model (Koutroumpa et al. 2007, 2009). The temperatures lower than $10^{5.64}$ are excluded by O vi emission observations (Shelton 2003) and interstellar ion absorption lines toward nearby stars.

On the other hand, it is difficult to fit with such a combination the Wisconsin data. In the UW (galactic center direction), a combination of hot gas and SWCX emission gives $(B+C)$ intensities as well as $C / B$ ratios roughly compatible with the observed values for several different temperature ranges. The main difficulty is the impossibility to account for the very low $C / B$ ratio measured toward the galactic anticenter direction with the present input models used in our study. We note that the high $B$ intensity is also clearly a problem for any hot gas solution, including the very high depletion hypothesis, as shown by the study of Bellm \& Vaillancourt (2005). The SWCX contribution, which hardens the spectra, reinforces this difficulty.

However, further investigation is required on the model's uncertainties in order to quantify their influence on the spectral hardness of SWCX emission. Further analysis of the hydrogenic ion approximation is needed, since hydrogenic ions tend to emit photons at higher energies following CX (since high- $n$ to ground transitions are generally allowed) than the multielectron ions considered here (because selection rules and more complicated atomic structure lead to more cascades before the final transition to ground). Therefore, it is likely that the model spectra have significantly less flux at low energies than they should. Given the steeply decreasing effective areas at lower energies (see Figure 1), this would help explain some of the discrepancies seen in the $C / B$ ratio.

Also, the fact that no distinction was made between the neutral targets $(\mathrm{H}$ or $\mathrm{He})$ in the $1 / 4 \mathrm{keV}$ calculations, would also have an effect on the spectral hardness, since there is an effect on the electron capture level (roughly proportional to $I_{n}{ }^{-1 / 2}$, where $I_{n}$ is the neutral target ionization potential), the capture level with He being somewhat lower than with $\mathrm{H}$. As noted in Section 5, the Wisconsin survey is more sensitive to this effect than ROSAT because of its observation geometry (i.e., the Wisconsin DW observations looked more directly through the He cone, where SWCX emission has a softer spectrum), again helping to explain some of the $C / B$ ratio anomaly. Finally, at the low energies of the $B$ band, more detailed calculations of the heliospheric and magnetospheric signals must be performed, especially the low-energy secondary SWCX emissions in the heliosheath and heliotail, i.e., subsequent recombinations of partially neutralized SW high ions.

The authors wish to thank Dan McCammon and Steve Snowden for comments and useful discussions on the ROSAT and Wisconsin surveys and band responses. The work presented has benefited from very useful discussions at the ISSI workshop "From the Outer Heliosphere to the Local Bubble" and at the "Local Bubble and Beyond II" meeting. We are extremely grateful to the anonymous referee for his attentive report and valuable comments which resulted in significantly improving the paper.

\section{REFERENCES}

Allen, C. W. 1973, Astrophysical Quantities (3rd ed.; London: Univ. London, Athlone Press)

Bellm, E. C., \& Vaillancourt, J. E. 2005, ApJ, 622, 959

Bhardwaj, A., et al. 2007, Planet. Space Sci., 55, 1135

Bloch, J. J., Jahoda, K., Juda, M., McCammon, D., Sanders, W. T., \& Snowden, S. L. 1986, ApJ, 308, L59

Bowyer, C. S., Field, G. B., \& Mack, J. F. 1968, Nature, 217, 32

Bregman, J. N., \& Lloyd-Davies, E. J. 2006, ApJ, 644, 167

Burrows, D. N., \& Mendenhall, J. A. 1991, Nature, 351, 629

Cox, D. P. 1998, Lecture Notes in Physics (Berlin: Springer), 506, 121

Cravens, T. E. 1996, Geophys. Res. Lett., 24, 105

Cravens, T. E. 2000, ApJ, 532, L153

Cravens, T. E., Robertson, I. P., \& Snowden, S. L. 2001, J. Geophys. Res., 106, 24883

Frisch, P. C., \& York, D. G. 1983, ApJ, 271, L59

Hasinger, G., Burg, R., Giacconi, R., Hartner, G., Schmidt, M., Trumper, J., \& Zamorani, G. 1993, A\&A, 275, 1

Henley, D. B., \& Shelton, R. L. 2008, ApJ, 676, 335

Herbstmeier, U., Mebold, U., Snowden, S. L., Hartmann, D., Butler Burton, W., Moritz, P., Kalberla, P. M. W., \& Egger, R. 1995, A\&A, 298, 606

Hurwitz, M., Sasseen, T. P., \& Sirk, M. M. 2005, ApJ, 623, 911

Indebetouw, R., \& Shull, J. M. 2004, ApJ, 607, 309 
Jelinsky, P., Vallerga, J. V., \& Edelstein, J. 1995, ApJ, 442, 653

Jenkins, E. B. 2002, ApJ, 580, 938

Kharchenko, V. 2005, AIPC, 774, 271

Kharchenko, V., \& Dalgarno, A. 2000, J. Geophys. Res., 105, 1854

Kharchenko, V., \& Dalgarno, A. 2001, ApJ, 554, L99

Koutroumpa, D., Acero, F., Lallement, R., Ballet, J., \& Kharchenko, V. 2007, A\&A, 475, 901

Koutroumpa, D., Lallement, R., Kharchenko, V., \& Dalgarno, A. 2009, Space Sci. Rev., 143, 217

Koutroumpa, D., Lallement, R., Kharchenko, V., Dalgarno, A., Pepino, R., Izmodenov, V., \& Quémerais, E. 2006, A\&A, 460, 289

Lallement, R. 1998, in in IAU Colloq. 166, The Local Bubble and Beyond, ed. D. Breitschwerdt, M. J. Freyberg, \& J. Truemper (Berlin: Springer-Verlag), 19

Lallement, R. 2004, A\&A, 422, 391

Lallement, R., Quémerais, E., Bertaux, J. L., Ferron, S., Koutroumpa, D., \& Pellinen, R. 2005, Science, 307, 1447

Lallement, R., Welsh, B. Y., Vergely, J. L., Crifo, F., \& Sfeir, D. 2003, A\&A, 411,447

Landi, E., Del Zanna, G., Young, P. R., Dere, K. P., Mason, H. E., \& Landini, M. 2006, ApJS, 162, 261

Lehner, N., Jenkins, E. B., Gry, C., Moos, H. W., Chayer, P., \& Lacour, S. 2003, ApJ, 595, 858

Lisse, C. M., et al. 1996, Science, 274, 205

McCammon, D., Burrows, D. N., Sanders, W. T., \& Kraushaar, W. L. 1983, ApJ, 269, 107

McMullin, D. R., et al. 2004, A\&A, 426, 885

Pepino, R., Kharchenko, V., Dalgarno, A., \& Lallement, R. 2004, ApJ, 617 , 1347

Quémerais, E., Lallement, R., Ferron, S., Koutroumpa, D., Bertaux, J.-L., Kyrölä, E., \& Schmidt, W. 2006, J. Geophys. Res., 111, 9114

Raymond, J. C., \& Smith, B.W. 1977, ApJS, 35, 419
Robertson, I. P., Collier, M. R., Cravens, T. E., \& Fok, M.-C. 2006, J. Geophys. Res. (Space Phys.), 111, 12105

Robertson, I. P., \& Cravens, T. E. 2003, Geophys. Res. Lett., 30, 1439

Sanders, W. T., Kraushaar, W. L., Nousek, J. A., \& Fried, P. M. 1977, ApJ, 217, L87

Sasseen, T. P., Hurwitz, M., Lisse, C. M., Kharchenko, V., Christian, D., Wolk, S. J., Sirk, M. M., \& Dalgarno, A. 2006, ApJ, 650, 461

Schwadron, N. A., \& Cravens, T. E. 2000, ApJ, 544, 558

Shelton, R. L. 2003, ApJ, 589, 261

Shull, J. M., \& Slavin, J. D. 1994, ApJ, 427, 784

Slavin, J. D., \& Frisch, P. C. 2002, ApJ, 565, 364

Smith, R. K., Brickhouse, N. S., Liedahl, D. A., \& Raymond, J. C. 2001, ApJ, 59,141

Smith, R. K., et al. 2007, PASJ, 556, L91

Snowden, S. L., Collier, M. R., \& Kuntz, K. D. 2004, ApJ, 610, 1182

Snowden, S. L., Cox, D. P., McCammon, D., \& Sanders, W. T. 1990, ApJ, 354, 211

Snowden, S. L., Egger, R., Finkbeiner, D. P., Freyberg, M. J., \& Plucinsky, P. P. 1998, ApJ, 493, 715

Snowden, S. L., Freyberg, M. J., Kuntz, K. D., \& Sanders, W. T. 2000, ApJS, 128,171

Snowden, S. L., Hasinger, G., Jahoda, K., Lockman, F. J., McCammon, D., \& Sanders, W. T. 1994, ApJ, 430, 601

Snowden, S. L., McCammon, D., Burrows, D. N., \& Mendenhall, J. A. 1994, ApJ, 424, 714

Snowden, S. L., Schmitt, J. H. M. M., \& Edwards, B. C. 1990, ApJ, 364, 118

Wargelin, B. J., Beiersdorfer, P., \& Brown, G. V. 2008, CaJPh, 86, 151

Welsh, B. Y., Crifo, F., \& Lallement, R. 1998, A\&A, 333, 101

Welsh, B. Y., \& Lallement, R. 2005, A\&A, 436, 615

Welsh, B. Y., \& Lallement, R. 2008, A\&A, 490, 707

Williamson, F. O., Sanders, W. T., Kraushaar, W. L., McCammon, D., Borken, R., \& Bunner, A. N. 1974, ApJ, 193, L133 